\title{
ULTRAMETRICS AND GEOMETRIC MEASURES
}

\author{
H. MOVAHEDI-LANKARANI AND R. WELLS
}

(Communicated by James E. West)

\begin{abstract}
Let $Z$ be a locally connected, locally compact, and separable metric space equipped with a geometric measure $\nu$. It is shown that if a subset $Y$ of $Z$ is bi-Lipschitz isomorphic to an ultrametric space, then $\nu(Y)=0$.
\end{abstract}

\section{INTRODUCTION AND RESULTS}

An $N$-dimensional ( $N>0$ a real number) geometric measure on a metric space $\left(Z, d_{Z}\right)$ is a Borel measure $\nu$ on $Z$ with the property that there exist constants $\rho \in(0, \infty]$ and $C \in[1, \infty)$ such that $r^{N} / C \leq \nu(B(z, r)) \leq C r^{N}$ for all $z \in Z$ and $r \in(0, \rho)$; cf. [3]. Here, $B(z, r)$ denotes the open ball of radius $r$ centered at $z$. In particular, the Lebesgue measure $\mu$ on $\mathbb{R}^{N}$ is an $N$-dimensional geometric measure. We note that if $Z$ is a metric space which admits a geometric measure $\nu$, then $Z$ does not have any isolated points. Moreover, $\nu(\{z\})=0$ for every $z \in Z$.

A map $f:\left(X, d_{X}\right) \rightarrow\left(Z, d_{Z}\right)$ of metric spaces is said to be bi-Lipschitz if there exists a constant $L \in[1, \infty)$ such that $d_{X}\left(x_{1}, x_{2}\right) / L \leq d_{Z}\left(f\left(x_{1}\right), f\left(x_{2}\right)\right)$ $\leq \operatorname{Ld}_{X}\left(x_{1}, x_{2}\right)$ for all $x_{1}, x_{2} \in X$. In this case we say that the spaces $X$ and $f(X)$ are bi-Lipschitz isomorphic. An important property of bi-Lipschitz maps is that they preserve Hausdorff dimension. In this paper we study the "size" of a subset $Y$ of a metric space $Z$, where every point of $Y$ has a neighborhood in $Y$ which is bi-Lipschitz isomorphic to an ultrametric space. Recall that a metric space $\left(X, d_{X}\right)$ is an ultrametric space if $d_{X}\left(x_{1}, x_{2}\right) \leq$ $\max \left\{d_{X}\left(x_{1}, x_{3}\right), d_{X}\left(x_{2}, x_{3}\right)\right\}$ for every $x_{1}, x_{2}, x_{3} \in X$. It is well known that ultrametric spaces are topologically "very small"; they are all of covering dimension zero. Yet there exist ultrametric spaces with nontrivial $N$-dimensional Hausdorff measure for $N>0$. Our main result implies that no such space can be bi-Lipschitz isomorphic to a subset of a locally connected metric space $Z$ equipped with an $N$-dimensional geometric measure.

Received by the editors November 3, 1993.

1991 Mathematics Subject Classification. Primary 54E40; Secondary 28A75, 28A78.

Key words and phrases. Lipschitz, ultrametric, geometric measure, Lebesgue measure, logarithmic ratio, Hausdorff dimension, metric dimension.

The second author was partially supported through grant N00014-90-J-4012 of the Office for Naval Research. 
Theorem 1.1. Let $N>0$ be a real number and let $Z$ be a locally connected metric space equipped with an $N$-dimensional geometric measure $\nu$. Let $Y$ be a subset of $Z$ with the property that every point of $Y$ has a neighborhood in $Y$ which is bi-Lipschitz isomorphic to an ultrametric space. Suppose that $Y$ is separable and that every point of $Y$ has a neighborhood in $Y$ whose closure in $Z$ is compact. Then $\nu(Y)=0$.

As an immediate corollary of the above theorem we get the following result.

Corollary 1.2. Let $Y$ be a subset of $\mathbb{R}^{N}$ with the property that every point of $Y$ has a neighborhood in $Y$ which is bi-Linschitz isomorphic to an ultrametric space. Then $\mu(Y)=0$.

The converse of Corollary 1.2 is obviously false. Indeed, a totally disconnected counterexample is provided by the set $Y=\{0\} \cup\left\{n^{-1} \mid n \geq 1\right\} \subset \mathbb{R}^{1}$; see [8, Proposition 2.14].

In order to state the next corollary, we need more terminology. A bi-Hölder equivalence of order $s>0$ (both above and below) $\left(X, d_{X}\right) \rightarrow\left(X^{\prime}, d_{X^{\prime}}\right)$ is a bi-Lipschitz isomorphism $\left(X, d_{X}^{s}\right) \rightarrow\left(X^{\prime}, d_{X^{\prime}}\right)$. The next result follows from the observation that if $\left(X, d_{X}\right)$ is an ultrametric space and $s>0$, then $\left(X, d_{X}^{s}\right)$ is also an ultrametric space.

Corollary 1.3. Let $Y$ be a subset of $\mathbb{R}^{N}$ with the property that every point of $Y$ has a neighborhood in $Y$ which is bi-Hölder equivalent of order $s>0$ to an ultrametric space. Then $\mu(Y)=0$.

In order to put the above two corollaries in a proper perspective, we observe that the Cantor ternary subset of $[0,1]$ is bi-Lipschitz isomorphic to an ultrametric space. However, if we require that $Y$ be locally isometrically isomorphic to an ultrametric space, then $Y$ must be even "smaller" - it must be locally finite and hence a discrete set. Indeed, an ultrametric space can be isometrically embedded in $\mathbb{R}^{N}$ if and only if it has at most $N+1$ points. This was independently proved by Timan [10] (in a special case), Lemin [5], and Aschbacher, Baldi, Baum, and Wilson [1]. In contrast, Kelly [4], Timan and Vestfrid [11], and Lemin [5] have independently shown that every ultrametric space can be isometrically embedded in a Hilbert space. More recently, Luukkainen and the first author [7] have established that any ultrametric space of metric dimension $<N$ can be bi-Lipschitz embedded in $\mathbb{R}^{N}$. (For definitions see [2] and [7].) They conjecture that no ultrametric space of metric dimension $N$ can be bi-Lipschitz embedded in $\mathbb{R}^{N}$. Corollary 1.2 settles this conjecture in the affirmative for a certain class of ultrametric spaces. (By [2, Proposition 8], the Hausdorff dimension of a metric space is less than or equal to its metric dimension.)

Corollary 1.4. Let $X$ be an ultrametric space of positive $N$-dimensional Hausdorff measure. Then there is no bi-Lipschitz embedding of $X$ into $\mathbb{R}^{N}$.

More generally, Semmes [9] has strengthened Corollary 1.4 to the following result.

Theorem 1.5 (S. Semmes). Let $X$ be an ultrametric space and suppose that there exists a bi-Lipschitz embedding $f$ of $X$ into $\mathbb{R}^{N}$. Then $f(X)$ has Hausdorff dimension less than $N$. 
In fact, we have learned that Luosto [6] has settled the conjecture in [7] in the affirmative. More specifically, he has established the following theorem.

Theorem 1.6 (K. Luosto). Let $X$ be an ultrametric space of metric dimension $\geq N$. Then there is no bi-Lipschitz embedding of $X$ into $\mathbb{R}^{N}$.

Finally, it was noted by the referee (with our thanks) that the proof of one of the results in an earlier version of this manuscript could be refined to prove the following proposition. (For a definition and some of the properties of logarithmic ratio see [8]. Also, see Section 2 for a definition of $\delta(\alpha)$.)

Proposition 1.7. Let $Y$ be a compact subset of $\mathbb{R}^{N}$ and let $R(Y)$ denote the logarithmic ratio of $Y$.

(a) If $R(Y)<1$, then $\mu(Y)=0$.

(b) If $R(Y)=1$ and if $I(r)<1$ for all $r>0$, then $\mu(Y)=0$. Here, $I(r)=\inf \{R(\alpha) \mid \alpha$ is a clopen partition of $Y$ with $\delta(\alpha)<r\}$.

\section{Proof OF THEOREM 1.1}

In the proof of Theorem 1.1 we may assume that $Y$ is compact and biLipschitz isomorphic to an ultrametric space. In fact, we may first assume that there is an ultrametric space $X$ with a bi-Lipschitz isomorphism $f: X \rightarrow Y$ and that $\bar{Y}$ is compact. Then it suffices to observe that the completion $\tilde{X}$ of $X$ is also ultrametric and that $f$ extends to a bi-Lipschitz isomorphism $\widetilde{X} \rightarrow \bar{Y}$, allowing us to replace $Y$ by $\bar{Y}$. It follows that $Y$ is totally disconnected. We may moreover assume that $Y$ is nondiscrete. Now Theorem 1.1 follows from Lemma 2.3 below.

Let $X$ be a nondiscrete totally disconnected compact metric space, and let $\mathscr{A}(X)$ be the set of all clopen partitions of $X$ different from the one-element partition $\{X\}$. If $\alpha \in \mathscr{A}(X)$, define the diameter

$$
\delta(\alpha)=\max \{\operatorname{diam} A \mid A \in \alpha\}>0
$$

of $\alpha$ and the gap

$$
\gamma(\alpha)=\min \left\{\operatorname{dist}\left(A, A^{\prime}\right) \mid A, A^{\prime} \in \alpha, A \neq A^{\prime}\right\}>0
$$

of $\alpha$. Then the set $\mathscr{A}^{*}(X)$ of sequences $\left(\alpha_{n}\right)$ in $\mathscr{A}(X)$ with $\lim _{n \rightarrow \infty} \delta\left(\alpha_{n}\right)=0$ is nonempty, and $\lim _{n \rightarrow \infty} \gamma\left(\alpha_{n}\right)=0$ for each $\left(\alpha_{n}\right) \in \mathscr{A}^{*}(X)$ by [8, Lemma 2.2]. In the proof of Lemma 2.3 we use the observation that exactly one of the following two properties holds:

(i) There is a sequence $\left(\alpha_{n}\right) \in \mathscr{A}^{*}(X)$ with $\gamma\left(\alpha_{n}\right)>\delta\left(\alpha_{n}\right)$ for all $n$.

(ii) Each sequence $\left(\alpha_{n}\right) \in \mathscr{A}^{*}(X)$ has a subsequence $\left(\alpha_{n_{k}}\right) \in \mathscr{A}^{*}(X)$ with $\gamma\left(\alpha_{n_{k}}\right) \leq \delta\left(\alpha_{n_{k}}\right)$ for all $k$.

Define $\mathscr{N}(Y, r)=\{z \in Z \mid \operatorname{dist}(z, Y)<r\}$ for $Y \subset Z$ and $r>0$.

Lemma 2.1. Let $Z$ and $\nu$ be as in Theorem 1.1, and let $Y$ be a nondiscrete totally disconnected compact subset of $Z$. Then there is a constant $\sigma>0$ with the following property: If $\alpha \in \mathscr{A}(Y)$ with $\delta(\alpha)<\sigma$ and if $s \in(0, \gamma(\alpha))$, then

$$
\nu\left(\mathcal{N}\left(Y, \frac{1}{2} \gamma(\alpha)\right)\right) \geq \nu\left(\mathcal{N}\left(Y, \frac{1}{2} s\right)\right)+\frac{\operatorname{card} \alpha}{4^{N} C}(\gamma(\alpha)-s)^{N} .
$$


Proof. Let $\mathscr{C}_{0}=\{D \mid D$ is a component of $Z\}$. Since $Z$ is locally connected, $\mathscr{C}_{0}$ is a clopen partition of $Z$. Hence, diam $D>0$ if $D \in \mathscr{C}_{0}$. Let $\mathscr{C}=\{D \in$ $\left.\mathscr{C}_{0} \mid D \cap Y \neq \varnothing\right\}$. Then $\alpha_{0}=\{D \cap Y \mid D \in \mathscr{C}\}$ is a clopen partition of $Y$.

Consider a parameter $\sigma>0$, which we adjust in the sequel, and a clopen partition $\alpha \neq\{Y\}$ of $Y$ with $\delta(\alpha)<\sigma$. Assuming $\sigma \leq \gamma\left(\alpha_{0}\right)$ if $\alpha_{0} \neq\{Y\}$, we have that $\alpha$ is a refinement of $\alpha_{0}$. If $D \in \mathscr{C}$, let $\alpha_{D}=\{A \in \alpha \mid A \subset D\}$ and $Y_{D}=\bigcup \alpha_{D}$; then $Y_{D}=Y \cap D$. We assume further that $\sigma \leq \min \{\operatorname{diam} D \mid D \in$ $\mathscr{C}\}$. We have that $Y_{D} \neq D$ whenever $D \in \mathscr{C}$, for if $Y_{D}=D$, then $D \in \alpha$ as $D$ is connected, and so $\delta(\alpha) \geq \operatorname{diam} D \geq \sigma$. Choose $z_{D} \in D \backslash Y_{D}$ if $D \in \mathscr{C}$. We assume that $\sigma$ is so small that $\frac{1}{2} \gamma(\alpha) \leq \min \left\{\operatorname{dist}\left(z_{D}, Y_{D}\right) \mid D \in \mathscr{C}\right\}$. We assume finally that $\sigma$ is so small that $\gamma(\alpha)<\rho$; then $\nu\left(\mathscr{N}\left(Y, \frac{1}{2} \gamma(\alpha)\right)\right)<\infty$ as $\mathscr{N}\left(Y, \frac{1}{2} \gamma(\alpha)\right) \subset \mathscr{N}\left(Y_{0}, \gamma(\alpha)\right)$ for some finite set $Y_{0} \subset Y$.

Let $s \in(0, \gamma(\alpha)), r=\frac{1}{4}(\gamma(\alpha)+s)$, and $r_{1}=\frac{1}{4}(\gamma(\alpha)-s)$. Consider $D \in \mathscr{C}$ and $A \in \alpha_{D}$. Since $r \leq \frac{1}{2} \gamma(\alpha) \leq \operatorname{dist}\left(z_{D}, Y_{D}\right) \leq \operatorname{dist}\left(z_{D}, A\right)$ and since $D$ is connected, there is a point $z_{A} \in D$ with $\operatorname{dist}\left(z_{A}, A\right)=r$. Let $B(A)=$ $B\left(z_{A}, r_{1}\right)$. From $r-r_{1}=\frac{1}{2} s$ we get that $B(A) \cap \mathscr{N}\left(A, \frac{1}{2} s\right)=\varnothing$ and from $r+r_{1}=\frac{1}{2} \gamma(\alpha)$ that $B(A) \subset \mathscr{N}\left(A, \frac{1}{2} \gamma(\alpha)\right)$. Since $\left\{\mathscr{N}\left(A, \frac{1}{2} \gamma(\alpha)\right) \mid A \in \alpha\right\}$ is a partition of $\mathscr{N}\left(Y, \frac{1}{2} \gamma(\alpha)\right)$, we conclude that $\{B(A) \mid A \in \alpha\}$ is a disjoint family in $\mathscr{N}\left(Y, \frac{1}{2} \gamma(\alpha)\right) \backslash \mathscr{N}\left(Y, \frac{1}{2} s\right)$. Hence,

$$
\begin{gathered}
\nu\left(\mathscr{N}\left(Y, \frac{1}{2} \gamma(\alpha)\right)\right)-\nu\left(\mathscr{N}\left(Y, \frac{1}{2} s\right)\right) \\
\geq \sum_{A \in \alpha} \nu(B(A)) \geq(\operatorname{card} \alpha) \frac{1}{C} r_{1}^{N} \\
\quad=\frac{\operatorname{card} \alpha}{4^{N} C}(\gamma(\alpha)-s)^{N} .
\end{gathered}
$$

Lemma 2.2. Let $Z, \nu, Y$, and $\sigma$ be as in Lemma 2.1, and let $\left(\alpha_{n}\right) \in \mathscr{A}^{*}(Y)$ be such that $\delta\left(\alpha_{n}\right) \leq M\left(\gamma\left(\alpha_{n}\right)-\gamma\left(\alpha_{n+1}\right)\right)$ for all $n$ with some constant $M>0$. Then $\nu(Y)=0$.

Proof. Let $\mathscr{N}_{n}=\mathscr{N}\left(Y, \frac{1}{2} \gamma\left(\alpha_{n}\right)\right)$. We may assume that $\nu\left(\mathscr{N}_{n}\right)<\infty$ and $\delta\left(\alpha_{n}\right)<\sigma$ for all $n$. Since $\left(\mathscr{N}_{n}\right)$ is a decreasing family of open sets of finite measure with intersection $Y$, we have that $\lim _{n \rightarrow \infty} \nu\left(\mathscr{N}_{n}\right)=\nu(Y)$. Since $\left(\operatorname{card} \alpha_{n}\right)\left(\gamma\left(\alpha_{n}\right)-\gamma\left(\alpha_{n+1}\right)\right)^{N} \leq 4^{N} C\left(\nu\left(\mathscr{N}_{n}\right)-\nu\left(\mathscr{N}_{n+1}\right)\right)$ for all $n$ by Lemma 2.1, we get that

$$
\lim _{n \rightarrow \infty}\left(\operatorname{card} \alpha_{n}\right)\left(\gamma\left(\alpha_{n}\right)-\gamma\left(\alpha_{n+1}\right)\right)^{N}=0 .
$$

If $n \geq 1$, choose a point $p_{A} \in A$ for $A \in \alpha_{n}$. Then

$$
Y \subset \bigcup_{A \in \alpha_{n}} B\left(p_{A}, 2 \delta\left(\alpha_{n}\right)\right) \text {. }
$$

Hence,

$$
\nu(Y) \leq\left(\operatorname{card} \alpha_{n}\right) C 2^{N} \delta\left(\alpha_{n}\right)^{N} \leq C 2^{N} M^{N}\left(\operatorname{card} \alpha_{n}\right)\left(\gamma\left(\alpha_{n}\right)-\gamma\left(\alpha_{n+1}\right)\right)^{N} \rightarrow 0
$$

as $n \rightarrow \infty$, implying that $\nu(Y)=0$.

Lemma 2.3. Theorem 1.1 holds if the subset $Y$ is nondiscrete, compact, and bi-Lipschitz isomorphic to an ultrametric space. 
Proof. Assume first that there is a sequence $\left(\alpha_{n}\right) \in \mathscr{A}^{*}(Y)$ such that, letting $\delta_{n}=\delta\left(\alpha_{n}\right)$ and $\gamma_{n}=\gamma\left(\alpha_{n}\right)$, we have $\gamma_{n}>\delta_{n}$ for all $n$. Passing to a subsequence of $\left(\alpha_{n}\right)$ we may then assume that $\gamma_{n+1} \leq \gamma_{n}-\delta_{n}$ for all $n$. Now $\delta_{n} \leq \gamma_{n}-\gamma_{n+1}$ for all $n$. Hence $\nu(Y)=0$ by Lemma 2.2.

Consider then the remaining case. We are given an ultrametric space $\left(X, d_{X}\right)$, a homeomorphism $f: X \rightarrow Y$, and a constant $L \geq 1$ such that $d_{X}\left(x_{1}, x_{2}\right) / L \leq$ $d_{Z}\left(f\left(x_{1}\right), f\left(x_{2}\right)\right) \leq L d_{X}\left(x_{1}, x_{2}\right)$ for all $x_{1}, x_{2} \in X$. For $s \in(0$, $\operatorname{diam} X)$, let $\beta(s)$ be the cover of $X$ by closed balls of radius $s$. It is easily seen that any two such balls are either disjoint or identical. Consequently, $\beta(s)$ is a clopen partition of $X$ distinct from $\{X\}$ with $\delta(\beta(s)) \leq s$. Furthermore, it is also easy to see that the distance between any two disjoint closed $s$-balls is greater than $s$, implying that $\gamma(\beta(s))>s$.

Next, we define a clopen partition $\alpha(s) \neq\{Y\}$ of $Y$ by setting $\alpha(s)=$ $\{f(B) \mid B \in \beta(s)\}$. Then $\delta(\alpha(s)) \leq L \delta(\beta(s)) \leq L s$ and $\gamma(\alpha(s)) \geq \gamma(\beta(s)) / L \geq$ $s / L$, implying that $\delta(\alpha(s)) \leq L^{2} \gamma(\alpha(s))$.

Now choose an arbitrary sequence $\left(s_{n}\right)$ of positive numbers converging to zero and put $\alpha_{n}=\alpha\left(s_{n}\right), \delta_{n}=\delta\left(\alpha_{n}\right)$, and $\gamma_{n}=\gamma\left(\alpha_{n}\right)$; then $\left(\alpha_{n}\right) \in \mathscr{A}^{*}(Y)$ and $\delta_{n} \leq L^{2} \gamma_{n}$. Passing to a subsequence of $\left(s_{n}\right)$ we may assume that $\gamma_{n} \leq \delta_{n}$ for all $n$. Now passing again to a subsequence of $\left(s_{n}\right)$ we may assume, in addition, that $\delta_{n+1} \leq \delta_{n} / 2 L^{2}$ for all $n$. Then $\delta_{n} \leq 2 \delta_{n}-2 L^{2} \delta_{n+1} \leq 2 L^{2}\left(\gamma_{n}-\gamma_{n+1}\right)$. Hence $\nu(Y)=0$ by Lemma 2.2 .

\section{ACKNOWLEDGMENT}

We would like to thank the referee for simplifying and shortening our original proof (which used the concept of logarithmic ratio explicitly). We are also grateful for his help in revising this paper.

\section{REFERENCES}

1. M. Aschbacher, P. Baldi, E. B. Baum, and R. M. Wilson, Embeddings of ultrametric spaces in finite dimensional structures, SIAM J. Algebraic Discrete Methods 8 (1987), 564-577.

2. P. Assouad, Étude d'une dimension métrique liée à la possibilité de plongements dans $\mathbb{R}^{N}$, C. R. Acad. Sci. Paris Sér. A 288 (1979), 731-734.

3. A. Jonsson and H. Wallin, Function spaces on subsets of $\mathbb{R}^{N}$, Math. Rep. (Chur, Switzerland) (J. Peetre, ed.), vol. 2, pt. 1, Harwood Academic, New York, 1984.

4. J. B. Kelly, Metric inequalities and symmetric differences, Inequalities-II (O. Shisha, ed.), Academic Press, New York, 1970, pp. 193-212.

5. A. Yu. Lemin, Isometric imbedding of isosceles (non-Archimedean) spaces in Euclidean spaces, Dokl. Akad. Nauk SSSR 285 (1985), 558-562; English transl., Soviet Math. Dokl. 32 (1985), 740-744.

6. K. Luosto, private communication.

7. J. Luukkainen and H. Movahedi-Lankarani, Minimal bi-Lipschitz embedding dimension of ultrametric spaces, Fund. Math. 144 (1994), 181-193.

8. H. Movahedi-Lankarani, An invariant of bi-Lipschitz maps, Fund. Math. 143 (1993), 1-9.

9. S. Semmes, private communication.

10. A. F. Timan, On the isometric mapping of some ultrametric spaces into $L_{p}$-spaces, Trudy Mat. Inst. Steklov. 134 (1975), 314-326; English transl., Proc. Steklov Inst. Math. 134 (1975), 357-370. 
11. A. F. Timan and I. A. Vestfrid, Any separable ultrametric space can be isometrically imbedded in $\ell_{2}$, Funktsional. Anal. i Prilozhen 17 (1983), no. 1, 85-86; English transl., Functional Anal. Appl 17 (1983), 70-71.

Department of Mathematics, Penn State Altoona Campus, Altoona, Pennsylvania $16601-3760$

E-mail address: hml@math.psu.edu

Department of Mathematics, Penn State University, University Park, Pennsylvania 16802

E-mail address: wells@math.psu.edu 